\title{
$N$-水杨醋腙修饰 11-氮杂青蒿素衍生物的合成及性质研究
}

\author{
李四聪 ${ }^{a}$ 季绍聪 $b$ 赵亮 ${ }^{a}$ 廖晓雨 ${ }^{b}$ 刘传峰 ${ }^{a}$ \\ 汤浩东 ${ }^{a}$ 舒正宁 ${ }^{a}$ 杨 鹏*, $a$ 裴月湖 $*, b$ \\ ( ${ }^{a}$ 沈阳药科大学无涯学院 沈阳 110016) \\ ( ${ }^{b}$ 沈阳药科大学中药学院 沈阳 110016)
}

\begin{abstract}
摘要 合成了 $N$-水杨醛腙修饰的 11 -氮杂青蒿素及去氧类似物. 采用紫外、苂光、核磁共振以及分子模拟等技术分析 了二聚体 $(3 R, 5 \mathrm{a} S, 6 R, 8 \mathrm{a} S, 9 R, 12 S, 12 \mathrm{a} R)-11$ - $(((E)$-2-羟基-5-(4-差基-3-((E)- $(((3 R, 5 \mathrm{a} S, 6 R, 8 \mathrm{a} S, 9 R, 12 R, 12 \mathrm{a} R)-3,6,9-$ 三甲基-10氧代十氢-3,12-环氧[1,2]二氧杂 [4,3-i] 异喹啉-11(12H)-基)亚氨基)甲基)芐基)亚芐基)氨基)-3,6,9-三甲基十氢-3,12-环氧 [1,2]二氧杂[4,3-i]异喹啉-10(3H)-酮(3)的优势构像; 探索了它与血红素的作用行为; 研究了它与杯[4]味唑的结合、识别 及被增溶性能. 结果表明, 因水杨醛腙键的 $\mathrm{OH}$ 与席夫碱 $\mathrm{N}$ 原子之间的分子内氢键作用, 3 呈现分子钳状的优势构像, 使其得以识别血红素. 此外, 3 可被杯 $[4]$ 咔唑的疏水空腔封装, 且结合作用强(结合常数 $K_{\mathrm{a}}=2.8 \times 10^{5} \mathrm{~L} \cdot \mathrm{mol}^{-1}$ ), 因而其 水溶性得以改善, 这为今后生物活性测试奠定了基础.
\end{abstract}

关键词大环；分子识别；杯芳烃; 咔唑；青蒿素

\section{Syntheses and Property Evaluation of $\mathrm{N}$-Salicylaldehyde Hydrazone Modified 11-Azaartemisinins and Their Derivatives}

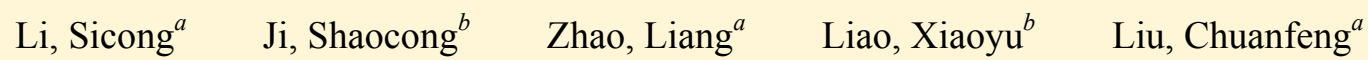 \\ Tang, Haodong ${ }^{a} \quad$ Shu, Zhengning ${ }^{a} \quad$ Yang, Peng $^{*, a} \quad$ Pei, Yuehu ${ }^{*, b}$ \\ $\left({ }^{a}\right.$ Wuya College of Innovation, Shenyang Pharmaceutical University, Shenyang 110016) \\ ( ${ }^{b}$ School of Traditional Chinese Materia Medica, Shenyang Pharmaceutical University, Shenyang 110016)
}

\begin{abstract}
N$-Salicylaldehyde hydrazone modified 11-azaartemisinins and their deoxy analogues were designed and synthesized. By using various techniques including UV-vis, fluorescence, NMR as well as molecular modeling, the conformations of $(3 R, 5 \mathrm{a} S, 6 R, 8 \mathrm{a} S, 9 R, 12 S, 12 \mathrm{a} R)-11-(((E)-2-h y d r o x y-5-(4-h y d r o x y-3-((E)-(((3 R, 5 \mathrm{a} S, 6 R, 8 \mathrm{a} S, 9 R, 12 R, 12 \mathrm{a} R)-3,6,9-$ trimethyl-10oxodecahydro-3,12-epoxy[1,2]dioxepino[4,3-i]isoquinolin-11(12H)-yl)imino)methyl)benzyl)benzylidene)amino)-3,6,9trimethyldecahydro-3,12-epoxy[1,2]dioxepino[4,3-i]isoquinolin-10(3H)-one (3) was analyzed, the binding behavior between 3 and hemin was measured, and the solubility-enhancing property of $\mathbf{3}$ by calix[4]carbazole was evaluated. The results show that the intramolecular hydrogen binding between the $\mathrm{N}$ atom of Schiff base in $\mathrm{N}$-salicylaldehyde hydrazone bond and the $\mathrm{OH}$ favors the formation of tweezer-like conformation of $\mathbf{3}$, which enables it to interact with hemin in $1: 1$ ratio. Moreover, the poor solubility of $\mathbf{3}$ in water could be enhanced due to its interaction with calix[4]carbazole, which pave the way for the further evaluation of the bioactivities of $\mathbf{3}$ in the future.
\end{abstract}

Keywords macrocycle; molecular recognition; carbazole; artemisinin

青蒿素(Artemisinin)是我国学者发现的一种含有内 酯环和过氧键的倍半萜类新型抗疮药物 ${ }^{[1]}$. 研究表明 ${ }^{[2]}$, 青蒿素的抗疮作用与疮原虫宿主红细胞中的血红素息 息相关. 在血红素催化作用下, 青蒿素过氧桥断裂, 形 成活性中间体. 这些中间体与疮原虫蛋白络合, 发生烷
基化作用, 从而杀伤㾏原虫. 多年来, 研究人员以青蒿 素的 10-位的酮羰基进行结构衍生合成出了多种药物, 如双氢青蒿素、蒿甲醚及青蒿琥酯等 ${ }^{[3]}$. 这些第一代药 物具有良好疮疾治疗效果, 然而也存在不足: 分子中缩 醛内酯键抗水解稳定性差, 口服给药生物利用度低; 此

* Corresponding authors. E-mail: yangpeng@syphu.edu.cn; peiyueh@vip.163.com

Received February 22, 2019; revised April 29, 2019; published online June 6, 2019.

Project supported by the Natural Science Foundation of Liaoning Province (No. 20180550874).

辽宁省自然科学基金(No. 20180550874)资助项目. 
外, 东南亚地区近年来出现对第一代青蒿素药物耐药病 例. 因此开发新型青蒿素药物极为迫切 ${ }^{[4]}$. 基于此, 人 们尝试对青蒿素其它位点进行衍生, 如 Singh 等 ${ }^{[5]}$ 对 11 位点进行修饰, 合成多种含 $N$-腙键的 11-氮杂青蒿素衍 生物, 对多药耐药性约氏疮原虫感染的小鼠展现出了良 好体内抗㾏疾活性. 此外, 该系列分子合成较为简单, 具有良好成药前景, 然而腙键在酸性条件下易水解性, 药物半衰期短, 血浆清除率高等不足是成药性研究中不 得不面对的问题.

与腙键相比, 水杨醛腙键因其 $\mathrm{C}=\mathrm{N}$ 键可与邻位 $\mathrm{OH}$ 形成分子内氢键, 稳定性得以极大提高. 另外, 孪 药(Twin drugs, 由两种相(不)同的药物经价键连接后而 成的新化合物)的设计思路也可以用来提高药物稳定性 和疗效 ${ }^{[6]}$. 基于以上两点, 本文合成了含 $N$-水杨醛腙键 的 11-氮杂青蒿素及其二聚体孪药, 研究了它们与血红
素靶点的识别及结合能力. 针对二聚体孪药水溶性差等 缺点, 研究了杯 [4]咔唑 ${ }^{[7]}$ 对其增溶性能. 这些分子水平 的研究为这类新型青蒿素衍生物的活性测试奠定了基 础.

\section{1 结果与讨论}

\section{1 化合物的合成及构型分析}

含水杨醛腙键的青蒿素及其去氧衍生物的合成路 线如 Scheme 1 所示. 首先, 参照文献[5]的方法合成了 中间体 $\mathbf{a}$ 和 $\mathbf{b}$, 然后合成目标化合物 $\mathbf{1} \sim \mathbf{4}$. 工艺条件为: 以苯为溶剂, Amberlyst-15 为催化剂, 室温下反应 $4 \mathrm{~h}$. 加入二氯甲烷后离心, 取上清液, 减压旋转蒸发除去溶 剂，最后采用硅胶柱进行分离得到. 其中，化合物 $\mathbf{3}$ 和 $\mathbf{4}$ 的收率较低(分别为 $12 \%$ 和 $13 \%$ ), 推测可能的原因是由 于这两个二聚体化合物溶解度差, 二氯甲烷不能良好溶 (i)<smiles>CC1C(=O)O[C@H]2O[C@@]3(C)CC[C@H]4[C@H](C)CC[C@@H]1[C@]42OO3</smiles>

$\stackrel{\mathrm{N}_{2} \mathrm{H}_{4} \cdot \mathrm{H}_{2} \mathrm{O}}{\longrightarrow}$<smiles>CC1C(=O)N(N)[C@H]2O[C@@]3(C)CC[C@H]4[C@@H](C)CC[C@@H]1[C@]42OO3</smiles>

中间体 $\mathbf{a}$<smiles>C[C@H]1CC[C@H]2[C@@H](C)C(=O)N(N)[C@@H]3O[C@]4(C)CC[C@@H]1[C@@]23CO4</smiles>

中间体 b

(ii)<smiles>CC1C(=O)N(N)[C@H]2O[C@@]3(C)CC[C@H]4[C@H](C)CC[C@@H]1[C@]42OO3</smiles>

中间体 $\mathbf{a}$

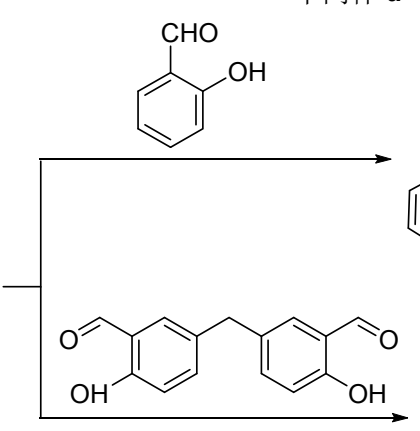

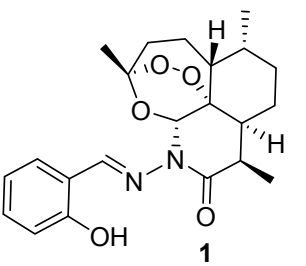

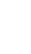
(1) 
解，造成损失. 化合物 $\mathbf{1} \sim \mathbf{4}$ 的结构均通过 ${ }^{1} \mathrm{H} N M R 、{ }^{13} \mathrm{C}$ NMR 和 HRMS 表征确认.

众所周知, 席夫碱类化合物存在顺反异构体. 本文 研究了含水杨醛腙键的青蒿素二聚体 $\mathbf{3}$ 的分子构型, 其 可能的构型列于图 1 中. 3 的局部 ${ }^{1} \mathrm{H}$ NMR 谱图(图 2a) 表明，该分子具有 $C_{2}$ 对称性，因此排除了构型 $\mathbf{A}$. 其二 维 NOESY 谱图(局部谱见图 $2 b$ ) 表明: $\mathrm{H}_{\mathrm{a}}$ 和 $\mathrm{H}_{\mathrm{b}}$ 存在明显 的 NOE 相关信号，因此可排除构型 $\mathbf{B}$ 的可能性. 所以, $\mathbf{c}$ 是化合物 $\mathbf{3}$ 的可能构型: 即水杨醛腙键的 $\mathrm{OH}$ 与席夫碱 $\mathrm{N}$ 原子之间形成分子内氢键. 采用 Spatan'14 分子软件的 半经验力场 $\mathrm{PM}_{3}$ 水平进行结构优化, 优化后 3 的最低能 量构像也表明(图 3): 水杨醛腙键中的 $\mathrm{OH}$ 与席夫碱 $\mathrm{N}$ 原子之间形成了分子内的氢键. 这一分子模拟结果与核 磁结果吻合. 除此之外, 从该图还可以看出, 3 呈现出一 种类似于分子钳的构像, 这促使我们进一步研究了该分 子对血红素的结合性能.
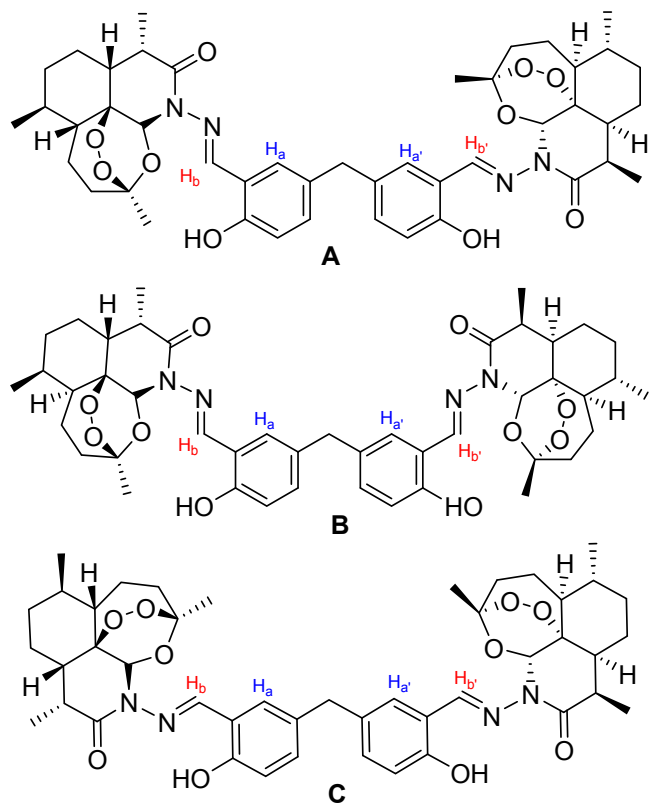

图 1 化合物 3 的可能构型

Figure 1 Possible configuration of $\mathbf{3}$

\section{2 青蒿素衍生物与血红素的分子识别研究}

紫外吸收光谱是研究主客体相互作用一种廉价、便 捷的常见测试方法. 一般说来, 主客体相互作用结果往 往会使吸收谱带的强度发生改变，同时伴随有红移或蓝 移现象的发生. 主客体发生有规律的结合时, 紫外吸收 图谱上会出现 “等吸收点” (又称等消光点). 因此，通过 紫外吸收光谱可以推测主客体结合模式、比例以及强度 等相关信息. 本文首先利用紫外滴定法研究 3 与血红素 (图 4)之间的相互作用(图 5). 结果表明, 滴加 3 对血红 素的紫外光谱产生了影响: $390 \mathrm{~nm}$ 处的吸收峰发生减色 红移, $405 \mathrm{~nm}$ 处出现明显等消光点, 表明 $\mathbf{3}$ 与血红素存
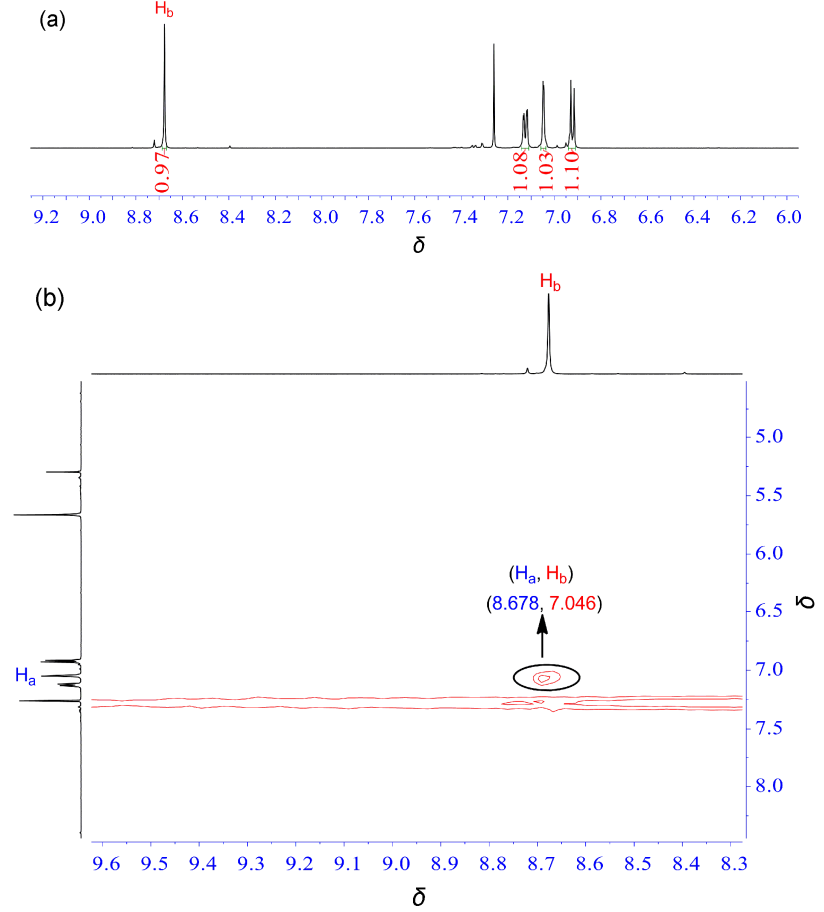

图 2 化合物 3 局部 ${ }^{1} \mathrm{H}$ NMR 谱图(a)及其 NOESY 的部分放大 谱图(b)

Figure 2 Partial ${ }^{1} \mathrm{H}$ NMR (a) and partial NOESY (b) of $\mathbf{3}$
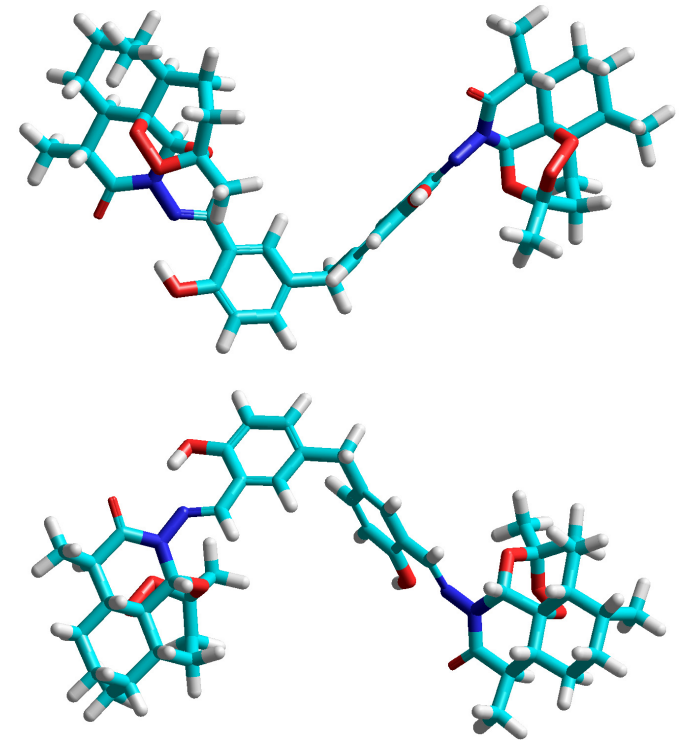

图 3 优化的化合物 $\mathbf{3}$ 能量最低构象

Figure 3 Optimized conformation of $\mathbf{3}$ with the lowest energy

在特异性结合模式. 采用 $1: 1$ 模型对这一变化进行拟 合, 相关系数为 0.97 , 证明该模型的有效性. JOB 曲线也 证实了两者的 $1: 1$ 结合模式(图 S14). 根据拟合结果, 求出两者结合常数为 $K_{\mathrm{a}}=1.02 \times 10^{5} \mathrm{~L} \cdot \mathrm{mol}^{-1}$.

为深入研究 3 与血红素的结合模式, 我们尝试了血 红素与 $1,2,4$ 的结合实验, 以作为对照. 然而, 去氧青 蒿素在水中溶解度要比青蒿素差很多, 在上述溶剂环境 中, 2 和 4 都不能溶解，因此无法测定与血红素的紫外滴 


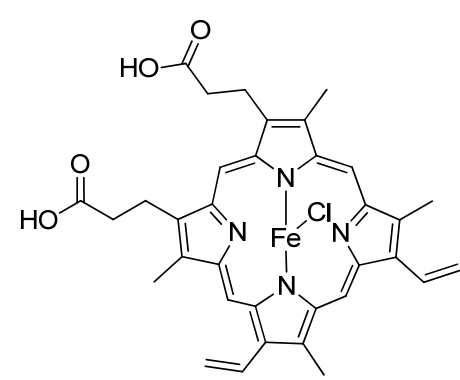

图 4 血红素分子结构

Figure 4 Molecular structure of hemin
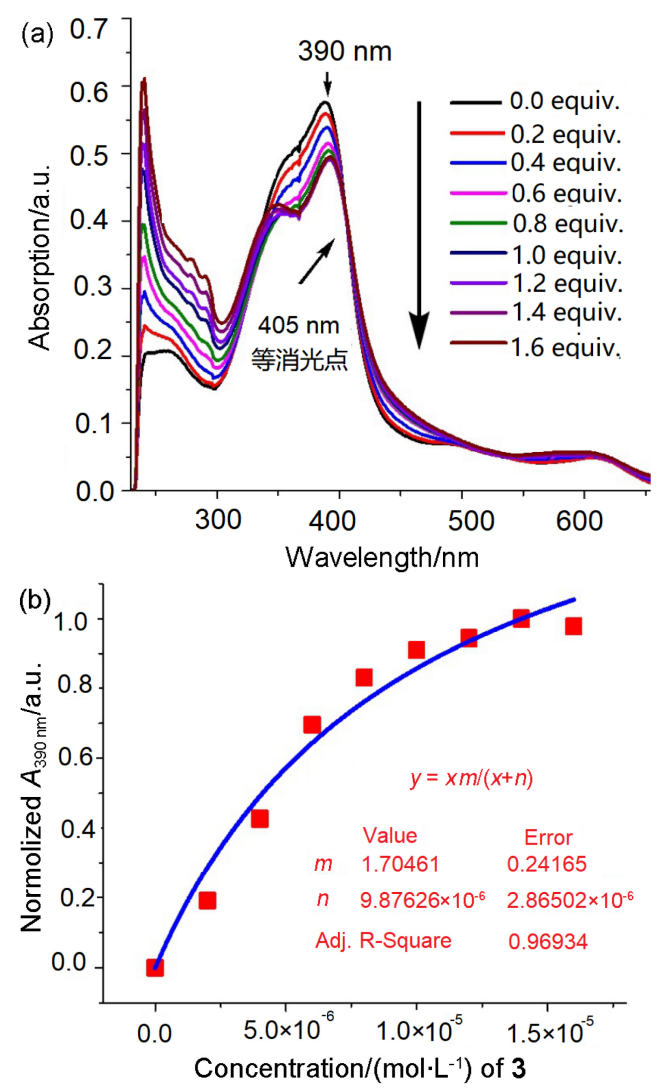

图 5 血红素 $(10 \mu \mathrm{mol} / \mathrm{L})$ 与 $\mathbf{3}$ 的紫外滴定图谱(a)及拟合曲线 (b)

Figure 5 UV-vis spectra (a) and the fitted curve (b) of hemin $(10 \mu \mathrm{mol} / \mathrm{L})$ upon addition of $\mathbf{3}$

定. 图 6 记录了血红素与 $\mathbf{1}$ 的紫外滴定谱图: 血红素在 $390 \mathrm{~nm}$ 处的紫吸收峰先下降后上升, 没有出现等消光 点, 表明 1 虽与血红素有所作用, 但并没有特异性的结 合模式. 这一结果反证了 3 与血红素特异性结合可能是 由于其自身钳形构像 ${ }^{[8]}$, 钳住了血红素所引起的(图 7).

由于血红素和 3 在当前环境下无苂光, 因此无法采 用苂光光谱法进一步研究两者的相互作用. 核磁共振虽 然也是研究分子相互作用的常见技术, 然而核磁检测通 常需要较高浓度的化合物(毫摩尔级别), 3 在这一浓度下 的水溶性极差, 这也限制了该方法的使用. 另外, 难溶 性也将影响到 3 的生物活性及成药性. 改进的方法有两

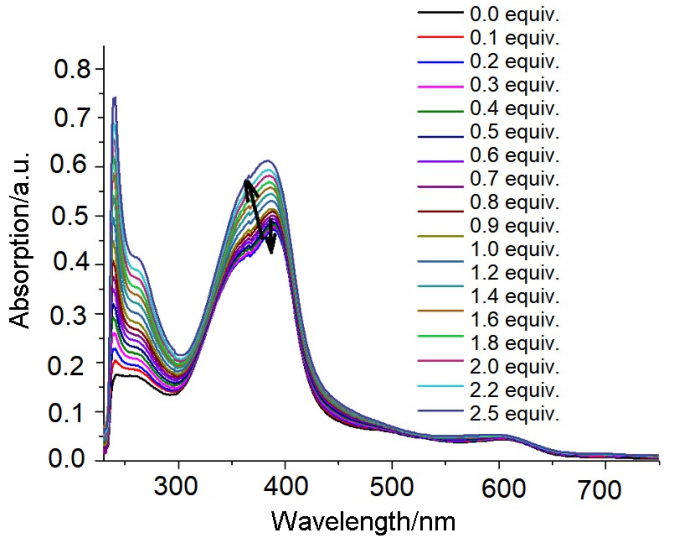

图 6 血红素 $(10 \mu \mathrm{mol} / \mathrm{L})$ 与 1 的紫外滴定图谱

Figure 6 UV-vis spectra of hemin upon addition of 1
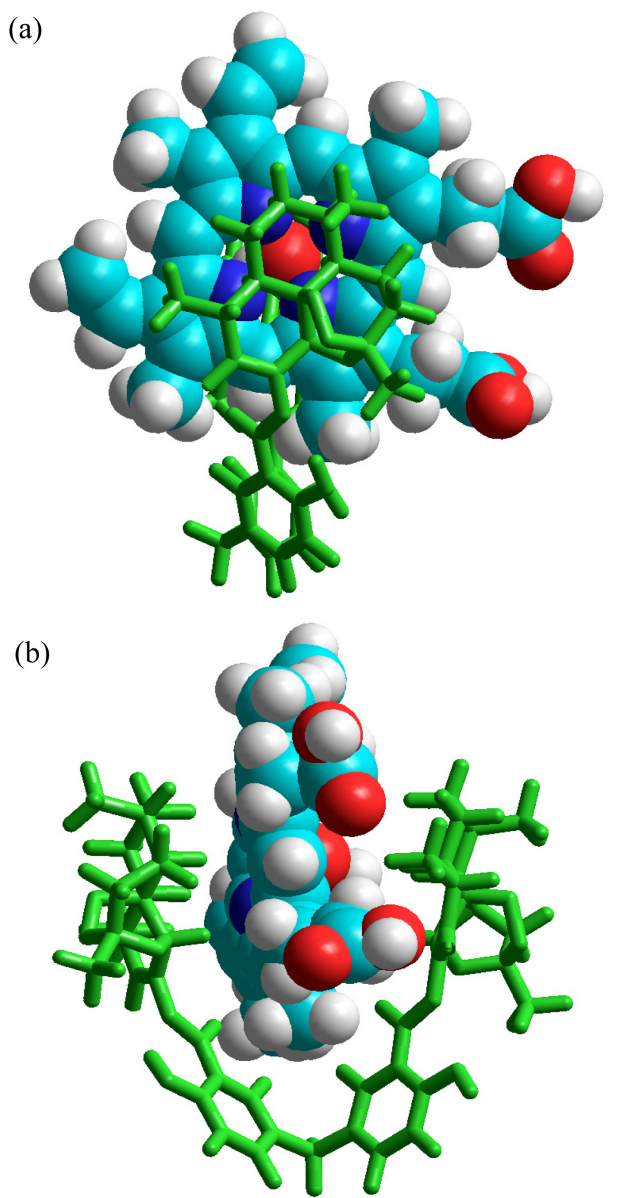

图 $7 \mathrm{MMFF}$ 力场优化的血红素与 $\mathbf{3}$ 的结合模式示意图

Figure 7 Optimized binding pattern of hemin with $\mathbf{3}$ (a) Front view, (b) side view

种, 一是对 3 进一步结构衍生, 引入水溶性官能团, 但 这需要繁琐的合成与分离工作; 另外一种方法是采用分 子载体对其进行增溶. 我们课题组在后一种方法具有一 定经验 ${ }^{[7 b]}$, 因此尝试此方案解决其水溶性问题.

\section{3 杯[4]卡唑对化合物 3 的识别及增溶作用}

杯 $[n]$ 咔唑是我们课题组 ${ }^{[7]}$ 报道的一类新型大环分 
子. 其中, 杯 [4]咔唑的疏水空腔可以封装、包载药物, 改善药物的水溶性(图 8). 因此本文研究了杯[4]咔唑对 3 的识别及增溶作用. 图 9 记录了两者相互作用的紫外 光谱. 图 9a表明: 当[3]/[杯咔唑] $<1.0$ 时, 杯 [4]咔唑 310 $\mathrm{nm}$ 处的吸收峰呈现减色红移现象, $303 \mathrm{~nm}$ 处出现一组

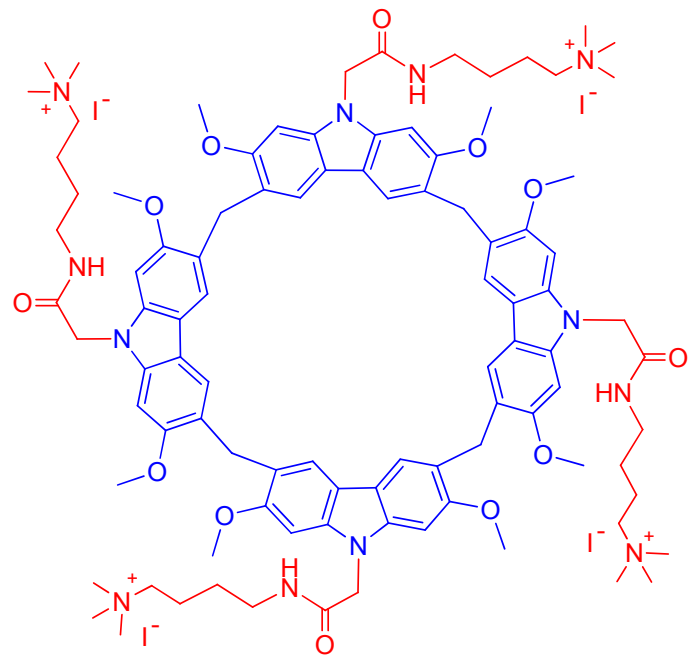

图 8 杯[4]咔唑结构示意图

Figure 8 Structure of calix[4]carbazole
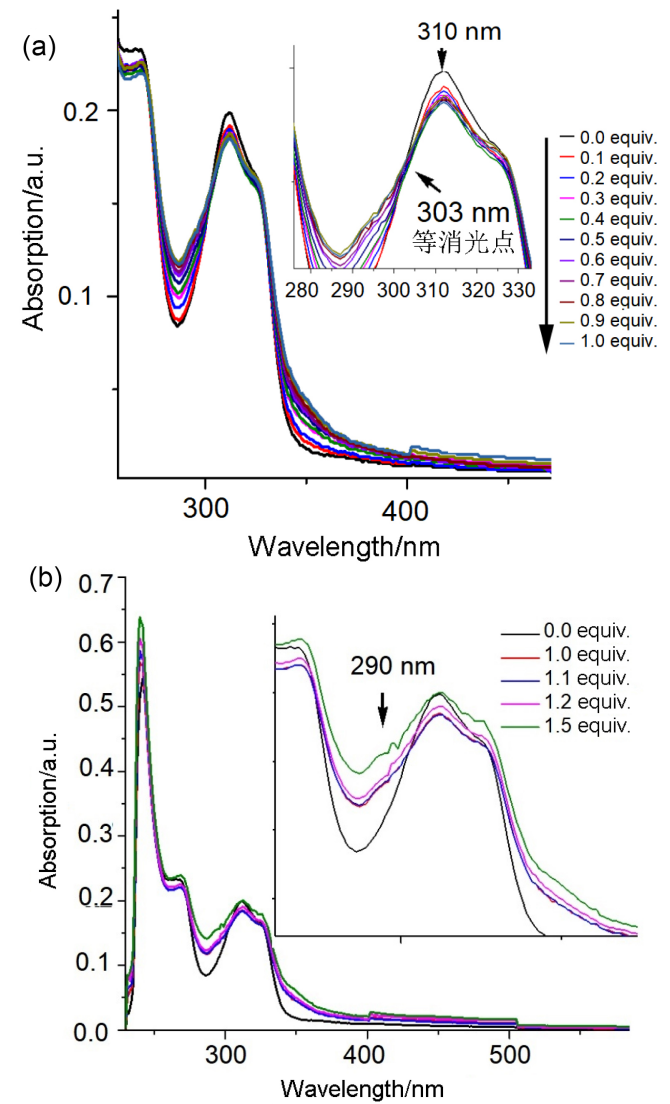

图 9 杯 [4]咔唑与 3 的紫外滴定谱图

Figure 9 UV-vis spectra of calix[4]carbazole upon addition of 3 (a) $[3] /[$ calixcarbazole $]<1.0$, (b) $[3] /[$ calixcarbazole $]>1.0$
等消光点，表明两者之间的特异性结合作用; 然而当 [3]/[杯咔唑] $>1.0,3$ 自身 $290 \mathrm{~nm}$ 处的吸收峰开始干扰 $310 \mathrm{~nm}$ 处吸收峰的走向(图 9b). 这种情况下, 很难采用 紫外滴定方式求出两者之间的结合能. 随后采用荧光光 谱进行了测试. 苂光滴定实验(图 10a)结果表明，随着 $\mathbf{3}$ 的不断加入, 杯咔唑的的荧光不断降低. 采用 $1: 1$ 模型 对结果进行了拟合(图 10b), $R^{2}$ 数值为 0.99 , 表明模型有 效. 两者 $1: 1$ 结合模式也被 $\mathrm{JOB}$ 曲线所验证, 求出结合

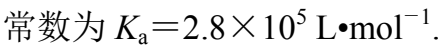
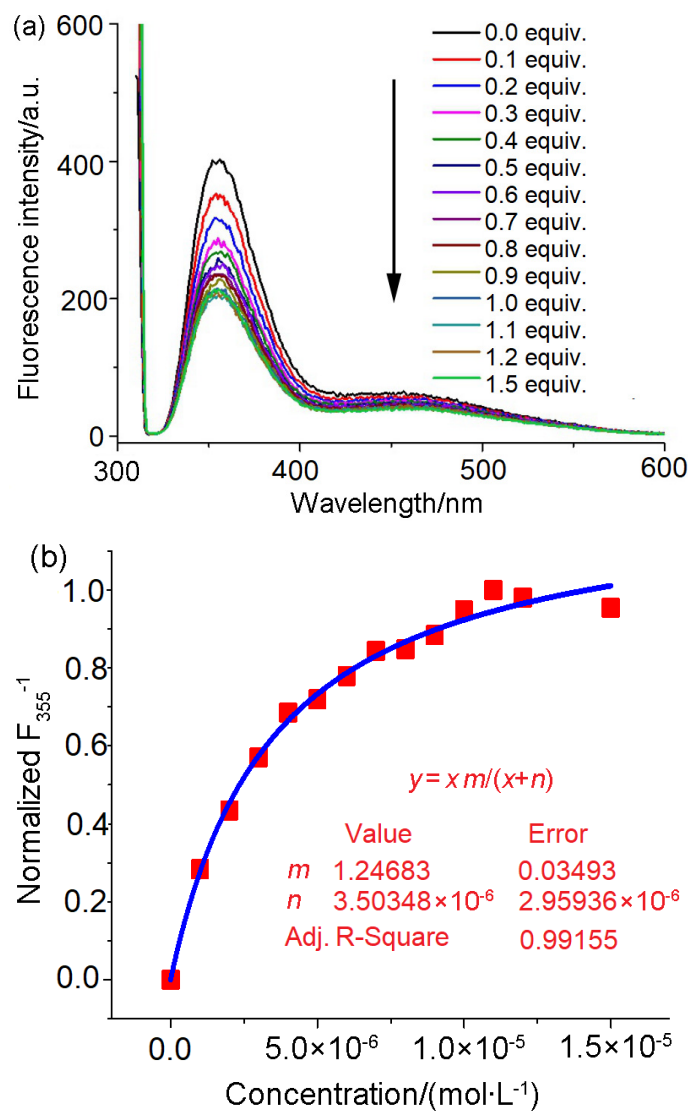

图 10 杯咔唑与 3 的苂光滴定谱图(a)及拟合曲线(b) Figure 10 Fluorescence spectra of calix[4]carbazole upon addition of $\mathbf{3}$ (a) and the fitted curve (b)

比较了 3 及其与杯咔唑复合物水溶性的差异，发现 杯咔唑的强结合作用可显著改善 $\mathbf{3}$ 的水中溶解度. 如图 11 所示，当没有杯咔唑存在时(图 11，右侧管), 3 自身的 溶解性能差, 有大量的固体析出. 作为对比, 当加入 1.5 倍量杯咔唑(图 11, 左侧管)时，此时观测不到明显的沉 淀，证明 3 的溶解性得到极大的改善. 这一增溶能力为 今后研究这些青蒿素衍生物的生物活性奠定了基础.

\section{2 结论}

设计、合成了含有 $N$-水杨醛酰腙键的新型青蒿素及 其去氧类生物; 研究了 3 的分子构型及其与血红素以及 


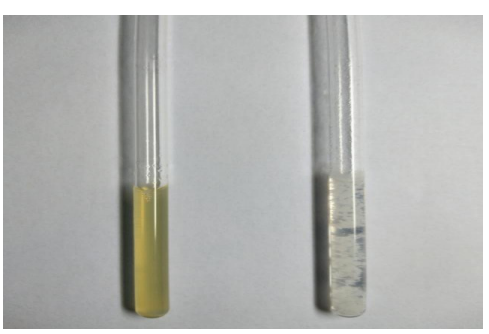

图 11 杯咔唑对 $\mathbf{3}$ 的增溶示意图(图左: 杯咔唑与 3 复合物; 图右: 3 自身)

Figure 11 Enhanced solubility of $\mathbf{3}$ by calix[4]carbazole (left: calix[4]carbazole/3 complexes; right: $\mathbf{3}$ alone)

杯[4]卡唑的结合性能. 结果表明: 3 可形成分子钳构像 结合血红素. 此外, 杯[4]咔唑能够识别和结合 3, 并增 进其水溶性, 表明其可作为分子载体, 为今后深入研究 青蒿素二聚体的生物活性奠定了基础.

\section{3 实验部分}

\section{1 仪器与试剂}

Bruker AVIII $600 \mathrm{MHz}$ 核磁共振仪, Bruker micro TOF_Q 质谱仪, Tu-1810 紫外-可见分光光度计, RF5301 荧光分光光度计, $H$ 型硅胶层析柱 $(200 \sim 300$ 目). 所用 试剂均为国产分析纯，使用前未经处理.

\section{2 合成方法}

$3.2 .1(3 R, 5 \mathrm{a} S, 6 R, 8 \mathrm{a} S, 9 R, 12 S, 12 \mathrm{a} R)-11-(((E)-2$ - 差基 5-(4- 差基-3- $((E)-(((3 R, 5 \mathrm{a} S, 6 R, 8 \mathrm{a} S, 9 R, 12 R, 12 \mathrm{a} R)-3,6,9-$ 三甲基-10-氧代十氢-3,12-环氧 [1,2]二氧杂 [4,3-i] 异喹 啉-11(12H)-基)亚氨基)甲基)芐基)亚苄基)氨基)-3,6,9三甲基十氢-3,12-环氧 [1,2] 二氧杂 [4,3-i]异喹啉10(3H) 酮(1)的合成

将 $290 \mathrm{mg}(0.98 \mathrm{mmol}$ )氮杂青蒿素(中间体 $\mathbf{a}$ )加入到 $3 \mathrm{~mL}$ 苯中溶解, 再加入 $300 \mathrm{mg}(2.45 \mathrm{mmol})$ 水杨醛和 30 $\mathrm{mg}$ Amberlyst-15, 搅拌, 室温反应 $4 \mathrm{~h}$, 反应结束后向反 应液中加入 $10 \mathrm{~mL}$ 二氯甲烷, 震荡离心, 取上清液, 减 压旋转蒸发, 除去溶剂, 硅胶柱分离(洗脱剂: 石油醚/ 乙酸乙酯, $V / V=15 / 1)$, 得到 $298 \mathrm{mg}$ 产品, 收率为 $76 \%$. $[\alpha]_{\mathrm{D}}^{25}-31.1\left(c 0.11, \mathrm{CHCl}_{3}\right)$, m.p. $52.0 \sim 54.3{ }^{\circ} \mathrm{C} ;{ }^{1} \mathrm{H}$ NMR (600 MHz, Chloroform- $d$ ) $\delta: 11.29(\mathrm{~s}, 1 \mathrm{H}), 8.37$ (s, $1 \mathrm{H}), 7.30(\mathrm{dt}, J=27.2,5.7 \mathrm{~Hz}, 2 \mathrm{H}), 6.99(\mathrm{~d}, J=8.1 \mathrm{~Hz}$, $1 \mathrm{H}), 6.91$ (t, $J=7.4 \mathrm{~Hz}, 1 \mathrm{H}), 5.67$ (s, 1H), 3.55 (p, $J=7.0$ $\mathrm{Hz}, 1 \mathrm{H}), 2.44(\mathrm{t}, J=12.3 \mathrm{~Hz}, 1 \mathrm{H}), 2.03(\mathrm{~d}, J=10.2 \mathrm{~Hz}$, $2 \mathrm{H}), 1.85(\mathrm{~d}, J=11.9 \mathrm{~Hz}, 1 \mathrm{H}), 1.82 \sim 1.71(\mathrm{~m}, 2 \mathrm{H}), 1.60 \sim$ $1.48(\mathrm{~m}, 2 \mathrm{H}), 1.42$ (td, $J=11.3,6.3 \mathrm{~Hz}, 1 \mathrm{H}), 1.34$ (s, 3H), $1.20(\mathrm{~d}, J=7.1 \mathrm{~Hz}, 3 \mathrm{H}), 1.11 \sim 0.99(\mathrm{~m}, 5 \mathrm{H}) ;{ }^{13} \mathrm{C}$ NMR (151 MHz, Chloroform- $d$ ) $\delta:$ 169.6, 162.5, 159.4, 132.2, 131.9, 119.1, 117.8, 117.2, 105.2, 81.1, 80.8, 51.4, 46.1, $37.5,36.5,34.3,33.6,25.4,25.0,22.9,19.7,12.5$. HRMS
(ESI/TOF-Q) calcd for $\mathrm{C}_{22} \mathrm{H}_{29} \mathrm{~N}_{2} \mathrm{O}_{5}[\mathrm{M}+\mathrm{H}]^{+}$401.2076, found 401.2072.

$3.2 .2(2 S, 4 \mathrm{a} S, 4 \mathrm{a} 1 R, 5 R, 7 \mathrm{a} S, 8 R, 10 \mathrm{a} R)-10-(((E)-2$ - 差 基 苯亚甲基) 氨基)-2,5,8-三甲基十氢 $-2,4 \mathrm{a} 1$ - 环氧杂 [4,3,2-ij] 异喹啉-9(2H)-酮(2)的合成

将 $280 \mathrm{mg}(1.00 \mathrm{mmol})$ 氮杂去氧青蒿素(中间体 b) 加入到 $3 \mathrm{~mL}$ 苯中溶解, 再加入 $300 \mathrm{mg}(2.45 \mathrm{mmol})$ 水杨 醛和 $30 \mathrm{mg}$ Amberlyst-15, 搅拌, 室温反应 $4 \mathrm{~h}$, 反应结 束后向反应液中加入 $10 \mathrm{~mL}$ 二氯甲烷, 震荡离心, 取上 清液, 减压旋转蒸发, 除去溶剂, 硅胶柱分离(洗脱剂: 石油醚/乙酸乙酯, $V / V=15 / 1)$, 得 $190 \mathrm{mg}$ 产品, 收率为 $49 \%$. $[\alpha]_{\mathrm{D}}^{25}+27.8\left(c 0.10, \mathrm{CHCl}_{3}\right)$, m.p. $53.0 \sim 55.1{ }^{\circ} \mathrm{C}$; ${ }^{1} \mathrm{H}$ NMR (600 MHz, Chloroform- $d$ ) $\delta: 8.50$ (s, 1H), 7.29 (d, $J=8.4 \mathrm{~Hz}, 1 \mathrm{H}), 7.00$ (d, $J=8.2 \mathrm{~Hz}, 1 \mathrm{H}), 6.91 \sim 6.85$ (m, 1H), $5.44(\mathrm{~s}, 1 \mathrm{H}), 3.32 \sim 3.28(\mathrm{~m}, 1 \mathrm{H}), 2.06(\mathrm{dt}, J=$ $13.3,4.3 \mathrm{~Hz}, 1 \mathrm{H}), 2.01 \sim 1.96(\mathrm{~m}, 1 \mathrm{H}), 1.91 \sim 1.87(\mathrm{~m}$, $1 \mathrm{H}), 1.80 \sim 1.85(\mathrm{~m}, 1 \mathrm{H}), 1.78 \sim 1.67(\mathrm{~m}, 2 \mathrm{H}), 1.47(\mathrm{~s}$, $3 \mathrm{H}), 1.40 \sim 1.33(\mathrm{~m}, 2 \mathrm{H}), 1.23(\mathrm{~d}, J=7.2 \mathrm{~Hz}, 3 \mathrm{H}), 1.14 \sim$ $1.07(\mathrm{~m}, 1 \mathrm{H}), 0.96 \sim 0.90(\mathrm{~m}, 5 \mathrm{H}) ;{ }^{13} \mathrm{C}$ NMR $(151 \mathrm{MHz}$, $\left.\mathrm{CDCl}_{3}\right) \delta: 168.3,158.9,151.3,131.5,131.3,118.8,118.0$, 117.3, 108.6, 85.1, 83.1, 45.9, 42.7, 35.2, 34.5, 33.9, 33.3, 24.2, 23.0, 22.2, 18.4, 12.2; HRMS (ESI/TOF-Q) calcd for $\mathrm{C}_{22} \mathrm{H}_{29} \mathrm{~N}_{2} \mathrm{O}_{4}[\mathrm{M}+\mathrm{H}]^{+}$385.2127, found 385.2130.

$3.2 .3(3 R, 5 \mathrm{a} S, 6 R, 8 \mathrm{a} S, 9 R, 12 S, 12 \mathrm{a} R)-11-(((E)-2$ - 羟基 5-(4- 羟基-3- $((E)-(((3 R, 5 \mathrm{a} S, 6 R, 8 \mathrm{a} S, 9 R, 12 R, 12 \mathrm{a} R)-3,6,9-$ 三甲基-10-氧十氢-3,12-环氧 [1,2] 二氧杂 [4,3-i] 异喹 啉-11(12H)-基)亚氨基)甲基)芐基)亚芐基)氨基)-3,6,9三甲基十氢 - 3,12 - 环氧 [1,2] 二氧杂 [4,3-i] 异喹啉$10(3 H)$-酮 $(3)$ 的合成

将 $1.20 \mathrm{~g}(4.04 \mathrm{mmol}$ )氮杂青蒿素(中间体 $\mathbf{a}$ )加入到 3 $\mathrm{mL}$ 苯中溶解, 再加入 $260 \mathrm{mg} 5,5^{\prime}$-亚甲基二(2-差基苯甲 醛) (1.01 mmol)和 $30 \mathrm{mg}$ Amberlyst-15, 搅拌, 室温反应 $4 \mathrm{~h}$. 反应结束后向反应液中加入 $10 \mathrm{~mL}$ 二氯甲烷, 震荡 离心, 取上清液, 减压旋转蒸发, 除去溶剂, 硅胶柱分 离(洗脱剂: 石油醚/乙酸乙酯, $V / V=10 / 1)$, 得 $95 \mathrm{mg}$ 产 品, 收率 $12 \% .[\alpha]_{D}^{25}-32.0\left(c 0.09, \mathrm{CHCl}_{3}\right)$, m.p. 42.2 $44.3{ }^{\circ} \mathrm{C} ;{ }^{1} \mathrm{H}$ NMR (600 MHz, Chloroform- $d$ ) $\delta: 8.68$ (s, $1 \mathrm{H}), 7.13(\mathrm{dd}, J=8.4,2.2 \mathrm{~Hz}, 1 \mathrm{H}), 7.05(\mathrm{~d}, J=2.2 \mathrm{~Hz}$, $1 \mathrm{H}), 6.92(\mathrm{~d}, J=8.4 \mathrm{~Hz}, 1 \mathrm{H}), 5.67(\mathrm{~s}, 1 \mathrm{H}), 3.86(\mathrm{~s}, 1 \mathrm{H})$, 3.54 (qd, $J=7.2,4.5 \mathrm{~Hz}, 1 \mathrm{H}), 2.46 \sim 2.41(\mathrm{~m}, 1 \mathrm{H}), 2.05 \sim$ $2.00(\mathrm{~m}, 2 \mathrm{H}), 1.83(\mathrm{dt}, J=13.3,3.5 \mathrm{~Hz}, 1 \mathrm{H}), 1.78 \sim 1.73$ (m, $2 \mathrm{H}), 1.57 \sim 1.50(\mathrm{~m}, 2 \mathrm{H}), 1.44 \sim 1.40(\mathrm{~m}, 1 \mathrm{H}), 1.33(\mathrm{~s}$, $3 \mathrm{H}), 1.19(\mathrm{~d}, J=7.2 \mathrm{~Hz}, 3 \mathrm{H}), 1.08 \sim 0.98(\mathrm{~m}, 5 \mathrm{H}) ;{ }^{13} \mathrm{C}$ NMR $\left(151 \mathrm{MHz}, \mathrm{CDCl}_{3}\right) \delta: 169.4,161.2,157.6,132.7$, 131.7, 117.6, 117.2, 105.1, 80.8, 80.7, 51.3, 45.9, 39.7, 
37.3, 36.4, 34.1, 33.5, 29.6, 25.3, 24.9, 22.7, 19.6, 12.3; HRMS (ESI/TOF-Q) calcd for $\mathrm{C}_{46} \mathrm{H}_{57} \mathrm{~N}_{4} \mathrm{O}_{12}[\mathrm{M}+\mathrm{COOH}]$ 857.3973, found 857.3991.

$3.2 .4(2 S, 4 \mathrm{a} S, 4 \mathrm{a} 1 R, 5 R, 7 \mathrm{a} S, 8 R, 10 \mathrm{a} R)-10-(((E)-2$-差基5-(4- 羟 基 -3-((E)-(((2R,4a $S, 4 \mathrm{a} 1 S, 5 R, 7 \mathrm{a} S, 8 R, 10 \mathrm{a} S)-2,5$, 8-三甲基-9-氧代十氢-2,4a1-环氧杂 [4,3,2-ij] 异喹啉10 (10a $H$ )-基)亚氨基) 甲基)芐基)亚芐基)氨基)-2,5,8三甲基十氢-2,4a1-环氧杂 [4,3,2-ij]异喹啉-9(2H)-酮 (4)的合成

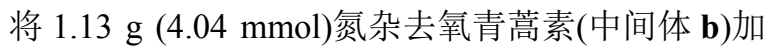
入到 $3 \mathrm{~mL}$ 苯中溶解, 再加入 $260 \mathrm{mg}$ 5,5'-亚甲基二(2-羟 基苯甲醛) (1.01 mmol)和 $30 \mathrm{mg}$ Amberlyst-15, 搅拌, 室 温反应 $4 \mathrm{~h}$, 反应结束后向反应液中加入 $10 \mathrm{~mL}$ 二氯甲 烷, 震荡离心, 取上清液, 减压旋转蒸发, 除去溶剂, 硅 胶柱分离(洗脱剂: 石油醚/乙酸乙酯, $V / V=10 / 1$ ), 得 $105 \mathrm{mg}$ 产品, 收率为 $13 \% .[\alpha]_{\mathrm{D}}^{25}+25.5\left(c 0.09, \mathrm{CHCl}_{3}\right)$, m.p. 44.3 46.0 ${ }^{\circ} \mathrm{C} ;{ }^{1} \mathrm{H}$ NMR (600 MHz, Chloroform- $d$ ) $\delta: 8.40$ (s, 1H), 7.12 (d, J=10.6 Hz, 1H), 7.05 (s, 1H), 6.95 $(\mathrm{d}, J=8.4 \mathrm{~Hz}, 1 \mathrm{H}), 5.42(\mathrm{~s}, 1 \mathrm{H}), 3.86(\mathrm{~s}, 1 \mathrm{H}), 3.31 \sim 3.27$ $(\mathrm{m}, 1 \mathrm{H}), 2.07 \sim 2.03(\mathrm{~m}, 1 \mathrm{H}), 1.99 \sim 1.95(\mathrm{~m}, 1 \mathrm{H}), 1.90 \sim$ $1.87(\mathrm{~m}, 1 \mathrm{H}), 1.83(\mathrm{dd}, J=13.9,5.1 \mathrm{~Hz}, 1 \mathrm{H}), 1.75(\mathrm{dd}, J=$ 13.4, $3.4 \mathrm{~Hz}, 1 \mathrm{H}), 1.72 \sim 1.67(\mathrm{~m}, 1 \mathrm{H}), 1.46(\mathrm{~s}, 3 \mathrm{H})$, $1.38 \sim 1.32(\mathrm{~m}, 2 \mathrm{H}), 1.23(\mathrm{~d}, J=7.2 \mathrm{~Hz}, 3 \mathrm{H}), 1.13 \sim 1.06$ $(\mathrm{m}, 1 \mathrm{H}), 0.96 \sim 0.90(\mathrm{~m}, 5 \mathrm{H}) ;{ }^{13} \mathrm{C} \mathrm{NMR}(151 \mathrm{MHz}$, $\left.\mathrm{CDCl}_{3}\right) \delta: 168.3,157.3,150.6,132.2,131.6,131.3,117.9$, 117.3, 108.6, 84.8, 83.2, 45.9, 42.7, 39.7, 35.2, 34.5, 33.9, 33.3, 24.1, 23.0, 22.2, 18.4, 12.2; HRMS (ESI/TOF-Q) calcd for $\mathrm{C}_{45} \mathrm{H}_{57} \mathrm{~N}_{4} \mathrm{O}_{8}[\mathrm{M}+\mathrm{H}]^{+}$781.4176, found 781.4136.

\section{3 荧光及紫外测试方法}

光谱测试环境均为: $10 \mathrm{mmol} / \mathrm{L} \mathrm{PBS}$ 缓冲溶液, $\mathrm{pH}=7.35$, 体系中含有 5\%二甲基亚砜(DMSO)以使化合 物完全溶解.
辅助材料(Supporting Information) 化合物 $1 \sim 4$ 的 ${ }^{1} \mathrm{H}$ $\mathrm{NMR} 、{ }^{13} \mathrm{C} \mathrm{NMR}$ 和 HRMS 等谱图. 这些材料可以免费 从本刊网站(http://sioc-journal.cn/)上下载.

\section{References}

[1] (a) Tu, Y. Y. Artemisia Annua and Artemisinin Drugs, Chemical Industry Press, Beijing, 2009 (in Chinese). (屠呦呦, 青蒿及青蒿素类药物, 化学工业出版社, 北京, 2009.) (b) Chang, Z. Sci. China Life Sci. 2016, 59, 81.

(c) Li, G. Q.; Li, Y.; Li, Z. L.; Zeng, M. Y. Artemisinin Antimalarial Drug, Science Press, Beijing, 2015 (in Chinese). (李国桥, 李英, 李泽琳，曾美怡，青蒿素类抗㾏药，科学出版 社, 北京, 2015.)

[2] (a) Eckstein, L. U.; Webb, R, J.; van Goethem, I. D. A.; East, J. M.; Lee, A. G.; Kimura, M.; O'Neill, P. M.; Bray, P. G.; Ward S. A.; Krishna, S. Nature 2003, 424, 957.

(b) O'Neill, P. M.; Posner, G. H. J. Med. Chem. 2004, 47, 2945.

(c) Utzinger, J.; Xiao, S. H.; Tanner, M.; Keiser, J. Curr. Opin. Invest. Drugs 2007, 8, 105

[3] You, Q. D. Medicinal Chemistry, 1st ed., Chemical Industry Press, Beijing, 2005, p. 402 (in Chinese). (尤启冬, 药物化学 (第一版), 化学工业出版社, 2005, p. 402)

[4] (a) Phyo, A. P.; Nkhoma, S.; Stepniewska, K.; Ashley, E. A.; Nair, S.; McGready, R.; ler Moo, C.; Al-Saai, S.; Dondorp, A. M.; Lwin, K. M.; Singhasivanon, P.; Day, N. P.; White, N. J.; Anderson, T. J.; Nosten, F. Lancet 2012, 379, 1960.

(b) Noedl, H.; Se, Y.; Schaecher, K.; Smith, B. L.; Socheat, D.; Fukuda, M. M. N. Engl. J. Med. 2008, 359, 2619.

[5] Singh, C.; Verma, V. P.; Hassam, M.; Singh, A. S.; Naikade, N. K.; Puri, S. K. J Med. Chem. 2014, 57, 2489.

[6] (a) Sun, Z. J. M.S. Thesis, Guangdong Pharmaceutical University, Guangzhou, 2008 (in Chinese). (孙政进, 硕士论文, 广东药学院, 广州, 2008.)

(b) Chen, Y. X.; Yu, P. L.; Li, Y.; Ji, R.Y. Acta Chim. Sinica 1985, 20, 470 (in Chinese).

(陈一心，虞佩琳，李英，嵇汝运，化学学报, 1985, 20, 470.)

[7] (a) Yang, Z.-Z.; Chen, Y.; Li, G.; Tian, Z.; Zhao, L.; Wu, X.; Ma, Q.; Liu, M.-Z.; Yang, P. Chem.-Eur. J. 2018, 24, 6087.

(b) Zhao, L.; Kang, L.; Chen, Y.; Li, G.; Wang, L.; Hu, C.; Yang, P. Spectrochim. Acta, Part A 2018, 193, 276.

(c) Yang, P.; Jian, Y.; Zhou, X.; Li, G.; Deng, T.; Shen, H.-Y.; Yang, Z.-Z.; Tian, Z.-M. J. Org. Chem. 2016, 81, 2974.

(d) Li, G.; Zhao, L.; Yang, P.; Yang, Z.; Tian, Z.; Chen, Y.; Shen, H.-Y.; Hu, C. Anal. Chem. 2016, 88, 10751

[8] Yao, H.; Sun, J. N.; Ke, H.; Yang, L. P.; Li, J. R. Jiang, W. Chin. J. Org. Chem. 2017, 37, 603 (in Chinese). (姚欢，孙娇囡，柯华，杨留攀，李加荣，蒋伟，有机化学，2017, 37, 603.) 